the sialidoses [2]. Investigators from University College, London, UK, and centers in Germany, examine the role of cerebellar pathology and alterations in inhibitory transmission in the pathogenesis of cortical myoclonus and ataxia [3].

\title{
References.
}

1. Lossos A, et al. JAMA Neurol. 2014 Jul 1;71(7):901-4.

2. Zupanc ML, Legros B. Progressive myoclonic epilepsy. Cerebellum. 2004;3(3):156-71.

3. Ganos C, et al. Mov Disord. 2014 Apr;29(4):437-43.

\section{ELECTROMYOGRAPHY AND METABOLIC MYOPATHIES}

Investigators at the Mayo Clinic, Rochester, $\mathrm{MN}$, report the sensitivity and specificity of the pediatric EMG in the diagnosis of myopathic disorders in patients $<18$ years referred between 2009 and 2013. Referral diagnoses included myopathy, muscle weakness, neuromuscular disorders, myositis, myalgia, myoglobinuria, myasthenia, myotonia, cramps, periodic paralysis, hypotonia, and developmental delay. Only patients with both EMG and muscle biopsy were included for analysis. Patients with neurogenic EMG and neuromuscular disorders were excluded.

Myopathic EMG was defined as short duration, low amplitude, polyphasic motor unit potentials with rapid recruitment. Of 72 patients included (age range, 6 months- 18 years), 32 had myopathic EMG with biopsy- or genetically-confirmed myopathy (Group A); 12 had myopathic EMG but normal biopsy (Group B); 3 had normal EMG but biopsy or genetically confirmed myopathy, all with metabolic myopathy (Group C); 25 had normal EMG and normal or nondiagnostic biopsy (Group D). The most common diagnoses were congenital myopathy ( 7 cases), metabolic myopathy (6 cases), muscular dystrophy (6 cases), genetically confirmed myopathy ( 5 cases), myopathy, undefined (5 cases), and inflammatory myopathy (4 cases). Pediatric EMG was $91 \%$ sensitive and $67 \%$ specific in myopathic disorders. Metabolic myopathies were commonly missed by EMG. (Ghosh PS, Sorenson EJ. Diagnostic yield of electromyography in children with myopathic disorders. Pediatr Neurol 2014 Aug;51(2):215-9).

COMMENTARY. Earlier studies show that the EMG has the highest concordance rate with neurogenic disorders but a lower concordance with myopathic disorders, especially in very young hypotonic infants [1]. More recent and the present studies show a higher rate of correlation in myopathic disorders but despite the exceptional expertise of the examiners, EMG frequently missed the diagnosis of a metabolic myopathy. Of 6 cases of metabolic myopathy, 3 had a normal EMG. Definitive diagnosis was made by muscle biopsy, biochemical analysis, and genetic testing of the muscle tissue. The metabolic abnormality was McArdle disease in 1 patient, mitochondrial myopathy in 1, mucolipidosis type II in 1, and undefined in 3 . The authors recommend that in cases presenting with a high degree of suspicion for metabolic myopathy, biopsy should be performed despite a normal EMG.

Electrophysiological Study of Mitochondrial Disorders. In 44 unselected mitochondrial disorder patients examined at the University of Pisa, Italy, motor nerve conduction studies were abnormal in $36.4 \%$, consistent with a sensori-motor axonal multifocal neuropathy, mainly affecting the lower limbs. EMG evidence of myopathy 
was present in $54.5 \%$ of patients. Peripheral nerve and muscle involvement is common but not uniformly present in mitochondrial disorders [2].

\section{References.}

1. Packer RJ, et al. Am J Dis Child. 1982 Dec;136(12):1057-9.

2. Mancuso M, et al. Neurol Sci. 2012 Apr;33(2):449-52.

\section{INFECTIOUS DISORDERS}

\section{THE RETINA AS A SURROGATE MARKER FOR PEDIATRIC CEREBRAL MALARIA}

Investigators from Malawi-Liverpool-Wellcome Trust Research Program, Malawi; University of Liverpool, UK; Michigan State University, USA; Vancouver General Hospital, Canada; and Ophthalmology Centers in Edinburgh, UK, review the evidence for associations between retina and brain neurovasculature, and the extent to which malarial retinopathy reflects cerebrovascular damage. Plasmodium falciparum is the causative organism in the majority of severe malaria cases, particularly in subSaharan Africa where children $<5$ years of age are disproportionately involved. Manifestations of severe pediatric malaria include convulsions, hypoglycemia, hyperparasitemia, coma, and malarial retinopathy. Funduscopic examination reveals white patchy discoloration of the macula, orange discoloration of retinal vessels, retinal hemorrhages with white centers, and papilledema. Associated abnormalities include metabolic acidosis and severe anemia. Duration of illness is short, and most patients either recover or die within $48 \mathrm{hrs}$. Patients who recover are at risk of neurologic disability and epilepsy.

Sequestration, resulting from binding of parasitized erythrocytes to vascular endothelium, is the hallmark of pediatric cerebral malaria, causing microvascular obstruction in both brain and retina. (MacCormick IJC, Beare NAV, Taylor TE, et al. Cerebral malaria in children: using the retina to study the brain. Brain 2014 Aug;137(Pt 8):2119-42).

COMMENTARY. This review illustrates the value of the funduscopic examination in the clinical diagnosis of neurologic disease. Despite some differences, the retinal pathology of microvasculature mimics the cerebral autopsy findings, and retinopathy is a surrogate marker for pediatric cerebral malaria. Both vascular beds are susceptible to sequestration of parasitized erythrocytes, leading to cerebral vascular obstruction, coma, convulsions, and neuropsychological sequelae.

\section{Cerebral Malaria Retinopathy Predictor of Neurocognitive Outcome.}

Investigators at Michigan State University, East Lansing; University of Michigan, Ann Arbor; Blantyre Malaria Project, Malawi; and Liverpool University Hospital, UK, studied the relationship of malaria-specific retinopathy during acute cerebral malaria to neurocognitive sequelae in 49 Malawian children tested 1 to 3 years following illness. Scores on Kaufman (mental processing), and TOVA (inattention and impulsivity) were worse in children with retinal hemorrhages, papilledema, optic disc hyperemia, whitening 